\title{
Biological Properties of HSC: Scientific Basis for HSCT
}

\author{
Alessandro Aiuti, Serena Scala, \\ and Christian Chabannon
}

\subsection{Introduction}

Hematopoiesis - from the Greek term for "blood making"-is the adaptive process by which mature and functional blood cells are continuously replaced over the entire lifetime of an individual. Erythrocytes, platelets, and the various subsets of leukocytes all have finite although different life spans. As a consequence, the daily production of red blood cells, platelets, and neutrophils in homeostatic conditions amount to more than 300 billion cells.

In mammals, after the emergence of the first hematopoietic progenitors in extra-embryonic structures such as the yolk sac in mice, cells of

A. Aiuti

San Raffaele Telethon Institute for Gene Therapy (SR-TIGET)/Pediatric Immunohematology and Bone Marrow Transplantation Unit, IRCCS Ospedale San Raffaele, Vita-Salute San Raffaele University, Milan, Italy

S. Scala

San Raffaele Telethon Institute for Gene Therapy (SR-TIGET), IRCCS Ospedale San Raffaele, Milan, Italy

C. Chabannon $(\varangle)$ Institut Paoli-Calmettes, Centre de Lutte Contre le Cancer, Marseille, France

Université d'Aix-Marseille, Marseille, France

Inserm BCT-1409, Centre d'Investigations Cliniques en Biothérapies, Marseille, France

e-mail: chabannonc@ipc.unicancer.fr

hematopoietic nature are first detected in the aorto-gonado-mesonephric (AGM) region of the developing embryo (Costa et al. 2012). The site of hematopoiesis then moves to the fetal liver and next to the BM where it remains established until the death of the individual. Extramedullary hematopoiesis in humans denotes a myeloproliferative syndrome.

The considerable knowledge accumulated over more than a century of experimental hematology led to the early understanding that all hematopoietic lineages are derived from a small subpopulation of undifferentiating and self-renewing stem cells. HSC represent the most accurately explored model of somatic stem cells that are present in most if not all tissues and organs, contributing to tissue homeostasis and repair. Existence of a population of HSC also has practical implications in terms of developing innovative therapies aiming at the definitive replacement or enhancement of a function in cells from one or several hematopoietic lineages, including the possibility to establish durable hematopoietic chimerism in recipients of allogeneic HSCT.

\subsection{Self-Renewal}

A general property of stem cells is self-renewal, assuming that when these cells divide, at least one of the "daughter cells" fully recapitulate the biological properties of the "mother stem cell." Self-renewal of the HSC population prevents 
exhaustion, while the hematopoietic tissue extensively proliferates and differentiates in steady-state conditions, as well as to repair various damages. Demonstration of self-renewal at the clonal level remains an arduous task, even though high-throughput analytical tools have been adapted. There is a growing body of evidence suggesting aging of the HSC population and decline of stem cell function with age (for a review, see Goodell and Rando 2015; de Haan and Lazare 2018). Appearance of "passenger mutations" in clonal hematopoiesis is one hallmark of aging (Cooper and Young 2017); the significance of such observations remains to be fully elucidated, but obviously raises questions when it comes to solicit elderly individuals to donate HSC for the benefit of a related patient.

\subsection{Commitment and Differentiation: New Data Challenge the Historical View of Hematopoietic Hierarchy}

The traditional view of HSC differentiation is a hierarchical representation of an inverted tree, where discrete and homogenous populations branch from one another, with successive restrictions in differentiation potentials. This oversimplifying view is increasingly challenged by recent studies reporting on noninvasive genetic experiments and clonal analyses in mice (for a review, see Laurenti and Göttgens 2018; Busch and Rodewald 2016). These studies suggest that hematopoietic differentiation uses different mechanisms under steady-state and stress conditions (Goyal and Zandstra 2015); however, both in steady-state conditions and transplantation models, only a small fraction of HSC contribute to long-term and stable reconstitution without compromising reestablishment of hematopoiesis (Schoedel et al. 2016; Höfer and Rodewald 2016), while most stem cells remain quiescent or proliferate infrequently. Single-cell transcriptional landscapes also suggest that differentiation occurs as a continuous rather than discrete physiological process and that restriction of differentiation is not the result of a "symmetric split" between the myeloid and lymphoid compartments as long thought through the phenotypic identification of "common myeloid progenitors" (CMP) and "common lymphoid progenitors" (CLP).

Commitment to one or several lineages, or conversely restriction in differentiation abilities, results from the expression of a controlled genetic and epigenetic program (Pouzolles et al. 2016; Antoniani et al. 2017; Gottgens, 2015); these mechanisms remain partially understood and thus can only be partially harnessed for in vitro engineering of HSC and their progeny (Rowe et al. 2016). The fate of HSC and their progeny is additionally regulated by extrinsic signals, among which hematopoietic growth factors and cytokines play an important role in survival, proliferation, and amplification (Kaushansky, 2006).

\subsection{The Bone Marrow Niches and Maintenance of Stemness (Fig. 7.1)}

Recent years have witnessed considerable progress in our understanding of organization and function of the bone marrow microenvironment. HSC establish interactions in the context of microanatomical organizations termed "niches." Progress has been made both in understanding the heterogeneity of niches at and within successive hematopoietic sites and in identifying various categories of cells - either of non-hematopoietic or of hematopoietic origin-that interact with HSC. The various types of bone marrow niches closely associate with the neurovascular network that infiltrates the central bone marrow as well as the endosteal region. The nature of the signaling between these different cell populations is also increasingly deciphered and involves many pathways, some of them unexpected at first (for a review, see Crane et al. 2017; Calvi and Link 2015). Replicating some of these interactions in vitro is key to successful expansion or genetic engineering of isolated HSC. Among the many molecular actors that govern interactions between HSC and the various cells present in niches, the CXCL12 chemokine and its most important 


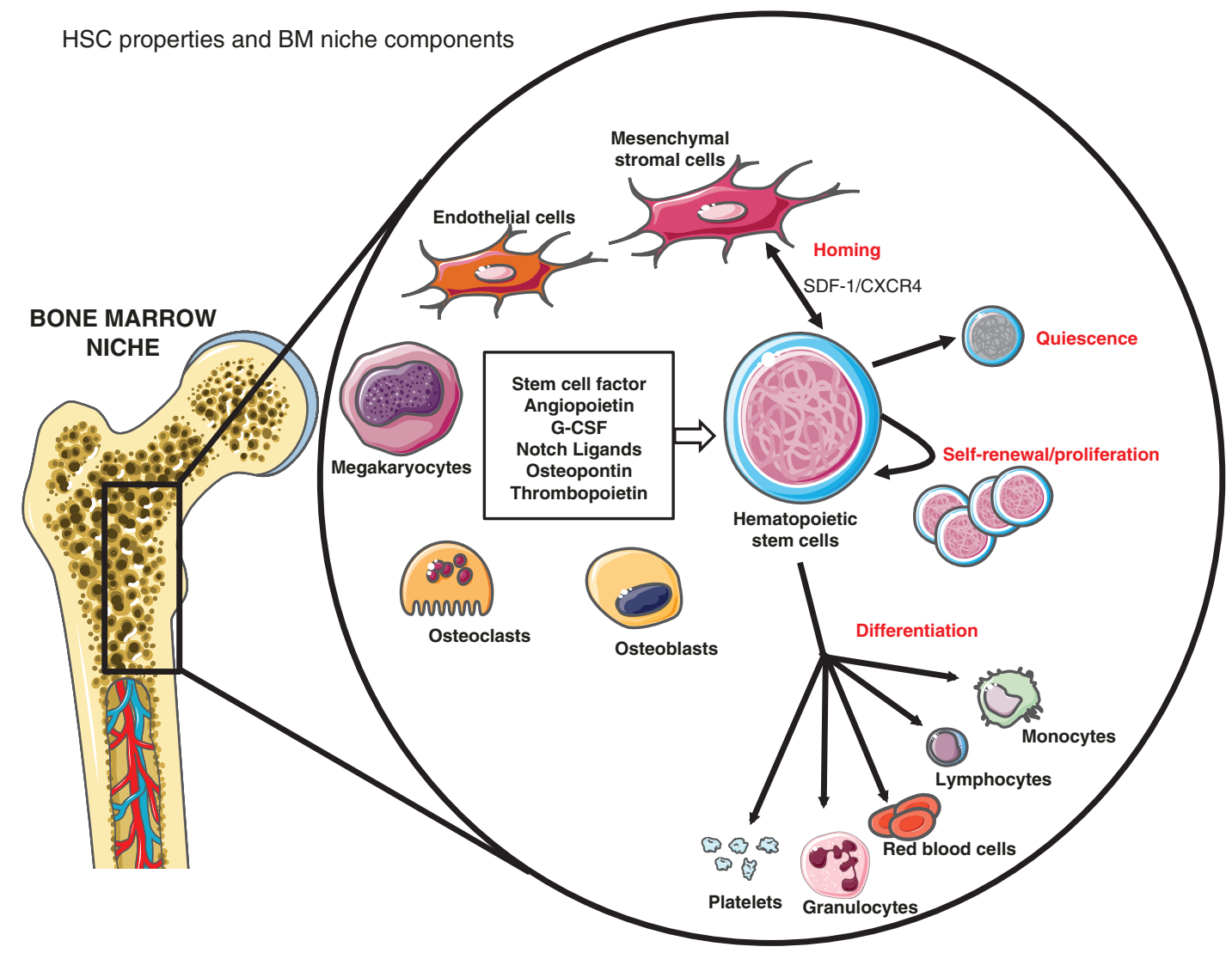

Fig. 7.1 HSC properties and BM niche components

receptor CXCR4 are of particular interest: direct or indirect modulation of this axis is clinically exploited to amplify the compartment of circulating stem cells that exist at low numbers in steadystate conditions.

\subsection{Preclinical Models of HSCT}

Most of the current knowledge on the biology of HSC and on therapeutic mechanisms of HSCT derives from studies in animal models (Eaves, 2015; Sykes and Scadden 2013). Classical murine transplantation studies showed that single or few engrafting HSC were sufficient and necessary to sustain long-term hematopoiesis in a reconstituted mouse. Human-in-mouse xenografts have become a fundamental tool to study hematopoietic dynamics upon HSCT. The generation of immune-deficient mice bearing a dele- tion of the interleukin-2 receptor gamma chain on the NOD-SCID background (NSG mice) was instrumental for studying HSC homing, engraftment, lineage differentiation, and serial transplantation capacity. This model has been further modified by introducing human myeloid cytokine genes to increase myeloid differentiation (Doulatov et al. 2012) or loss-of-function mutation in KIT receptor to efficiently support engraftment of human HSC without the need for conditioning therapy (Cosgun et al. 2014). To overcome the lack of human components in the murine BM, humanized-BM niche systems have been recently developed which are based on human stromal cells implanted on specific scaffold or directly injected with extracellular matrix to generate BM micro-ossicles (Di Maggio et al. 2011; Reinisch et al. 2016). These strategies provide novel tools to study the behavior of human HSC in their physiological context and to dissect 
the role of the niche upon transplantation. However, homing and engraftment parameters in xenografts may be different from the natural setting, and most HSCT models follow recipient mice for few months after transplantations, thus making long-term outcome difficult to assess.

Dogs provide an ideal preclinical modeling system for HSCT studies due to their large body size, life span, and high genetic diversity, which more appropriately recapitulate the human scenario. Preclinical canine modeling has been fundamental for the clinical translation of conditioning regimens and the importance of MHC donor/ recipient matching. However, the lack of canine reagents and the logistic difficulties of working with large animal models have precluded widespread availability (Stolfi et al. 2016). Auto-HSCT in nonhuman primates is arguably the experimental model most closely resembling humans; their treatment conditions - including the use of CD34+ cells, mobilization, and conditioning regimensall parallel those commonly exploited in human transplantation. While the ethical issues and costs have limited their use to selected centers, these animals are able to maintain long-term hematopoiesis up to several years after transplantation allowing the study of HSCT dynamics in a close-to-human fashion (Koelle et al. 2017).

\subsection{Gene Transfer/Gene Editing/ Gene Therapy Targeting HSC} (Fig. 7.2)

Ex vivo HSC gene therapy (GT) is based on the genetic modification of autologous HSC to correct monogenic disorders or to provide novel features to hematopoietic cells for treating infectious diseases or cancers (Naldini, 2011). It is now well established that HSC can be efficiently gene modified to continuously produce a cell progeny expressing the therapeutic gene while maintaining the ability to engraft long-term, for at least 15 years (Cicalese et al. 2016). Potential advantages of this approach over allogeneic HSCT include the lack of GVHD or rejection and the possibility of engineering HSC in order to achieve supra-physiological level of the corrected protein (Naldini, 2011; Aiuti and Naldini 2016).
Currently, integrating vectors derived from retroviruses represent the most efficient platform for engineering HSC and to provide permanent and heritable gene correction. $\gamma$-Retroviral vectors (RV) have been used in many clinical applications including GT of inherited blood disorders and cancer therapy. HSC-GT in primary immunodeficiencies was shown to provide clinical benefit, but the use of $\gamma$-RV was associated with risks of insertional mutagenesis due to activation of protooncogenes with the exception of ADA deficiency (Cicalese et al. 2016). Self-inactivating (SIN)lentiviral vectors (LV) are currently the tools of choice for most of the HSC-GT applications given their ability to transduce at higher efficiency nondividing cells, to carry larger and more complex gene cassettes, and to display a safer insertion site (InS) pattern in human HSC (Naldini, 2011). The recent development of designer endonucleases led to the advent of gene targeting approaches. In contrast to viral vectors, which can mediate only one type of gene modification (gene addition), genome-editing technologies can mediate gene addition, gene disruption, gene correction, and other targeted genome modifications (Dunbar et al. 2018). These strategies have the potential to overcome vector InS genotoxicity and to handle diseases due to dominant negative mutations. Despite the great promises, several challenges need to be addressed. Primitive, slow-cycling human BM-derived HSC are very resistant to ex vivo manipulations required for gene targeting, and the current efficiency of gene editing into repopulating HSC may not be suitable for clinical applications requiring high levels of correction (Dunbar et al. 2018; Kohn, 2017).

Thus, there remains a pressing need to develop methods to expand HSC or gene-corrected HSC while maintaining their repopulating capacity. Various cytokines and growth factors derived from BM niche, such as SCF, TPO, and Flt-3 ligand, are able to regulate HSC stemness and differentiation and are commonly used in HSC transduction protocols. However, even efficiently supporting GT, the balance between self-renewal/differentiation still hangs toward differentiation. High-throughput screening of chemical compounds has resulted in the identification of two promising molecules (StemRegenin1, SR1 (Wagner et al. 2016) and a 
Gene correction of HSC for cell-based therapies

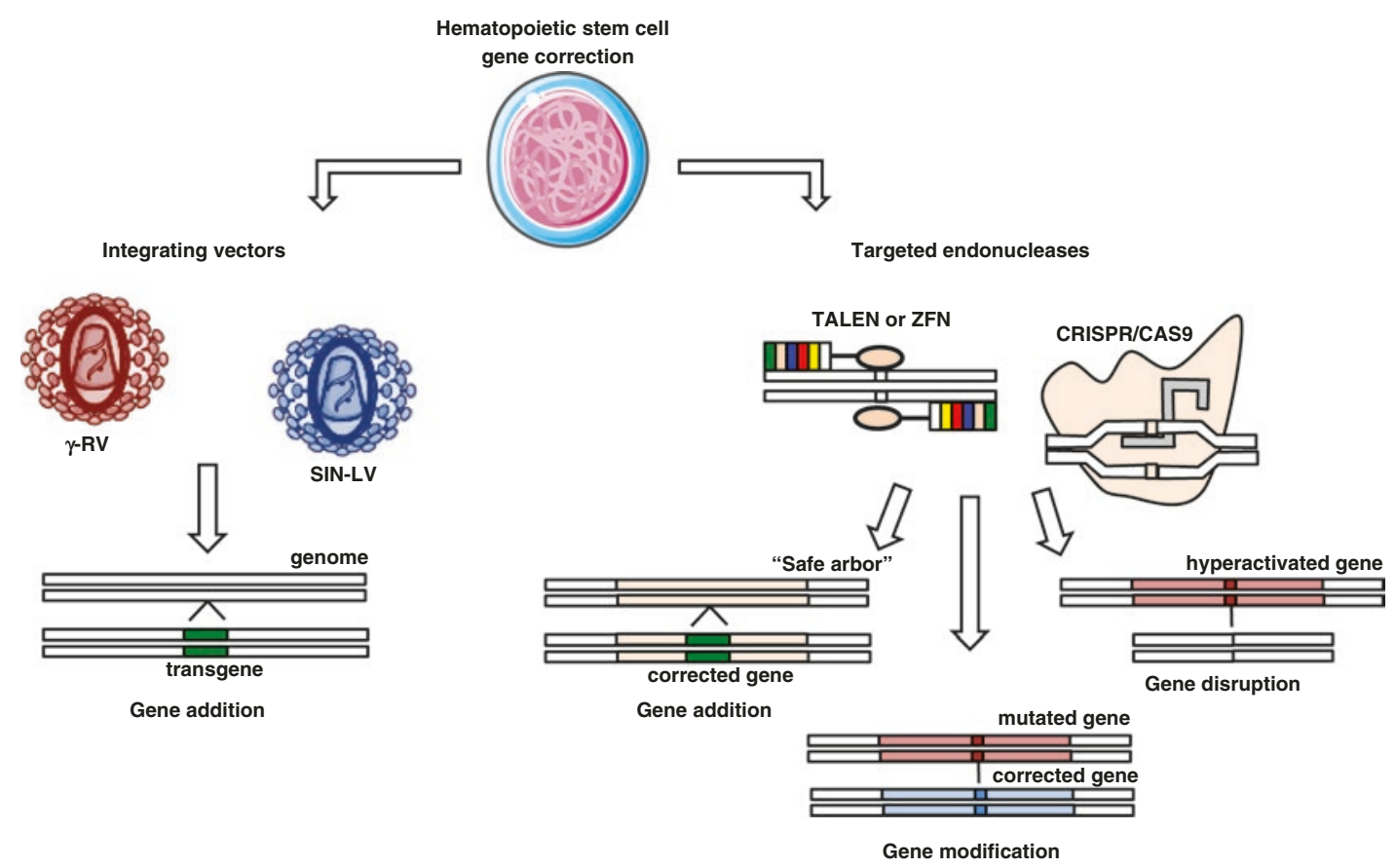

Fig. 7.2 Gene correction of HSC for cell-based therapies

pyrimidole derivative UM171 (Fares et al. 2014)) that are able to achieve great expansion of longterm repopulating HSC. Several small molecules have been identified that may support modest degrees of HSC expansion, but the ideal drug or combination has not yet been reported.

\subsection{Studying Dynamics \\ of Hematopoietic \\ Reconstitution upon \\ HSCT (Fig. 7.3)}

Upon gene correction, each transduced cell and its progeny become univocally marked by a specific insertion site (InS). The analysis of RV or LV InS has emerged as one of the most effective strategies allowing tracing the activity of genetically engineered hematopoietic cells directly in vivo in animal models as well as in GT-treated patients. Retrieving InS from mature blood cells after HSCT allowed studying the kinetics of blood cell production from individual stem cells within a heterogeneous population (Scala et al. 2016).
In the murine setting, the finding that the vast majority of the InS after transplant were present in either lymphoid or myeloid cells with few InS shared by both lineages led to the concept that murine HSC are heterogeneous and already biased for their fate. The possibility to directly translate these models on human beings is currently under investigation (Lu et al. 2011; Yamamoto et al. 2013).

Clonal tracking studies in nonhuman primates have been pivotal in studying HSCT dynamics in an experimental setting close to humans. The results of these works showed common pattern of hematopoietic reconstitution upon transplantation: clonal fluctuation in the early phases postHSCT, potentially due to the initial contribution to the hematopoiesis of short-term unilineage progenitors, followed by a recovery of a stable hematopoietic output likely related to the takeover of long-term multipotent HSC contribution. Thus, differently from murine studies, long-term HSC are able to provide multi-lineage engraftment, and there is no evidence of predetermined lineage choice at stem cell level in primates (Koelle et al. 2017; Kim et al. 2014). 


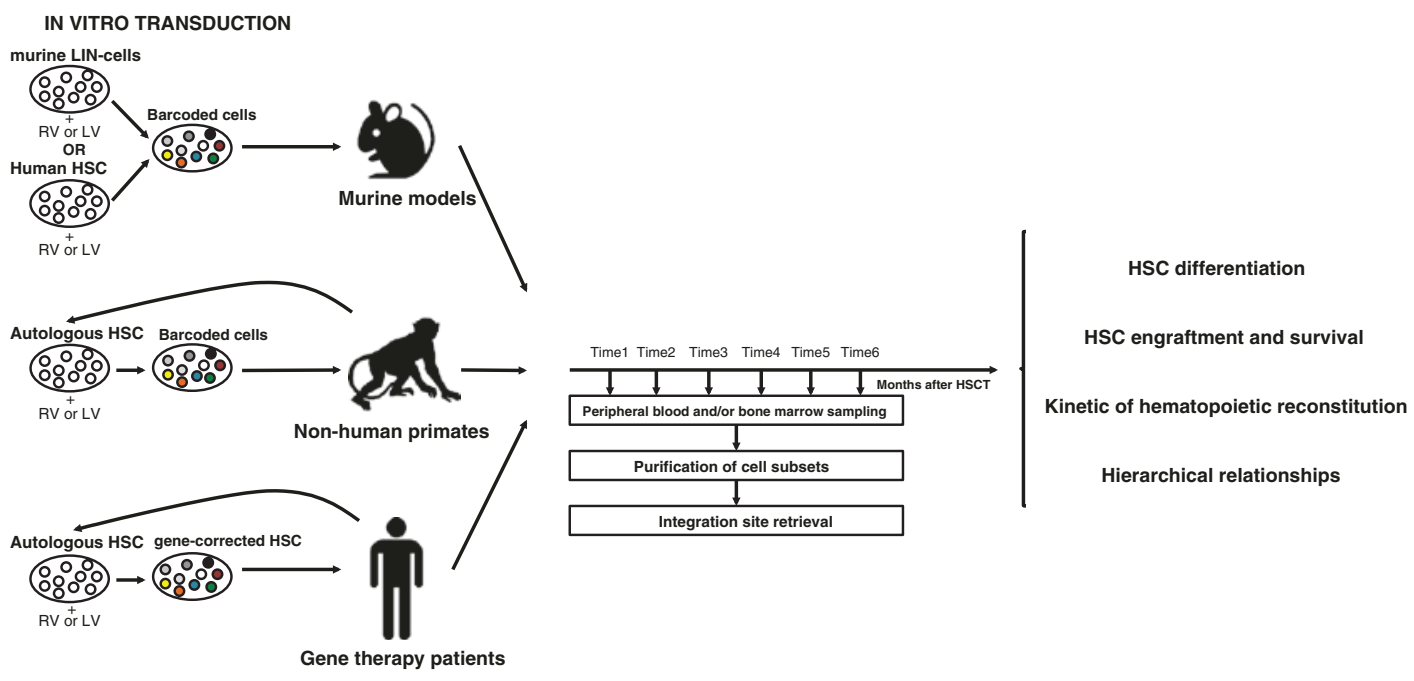

Fig. 7.3 Clonal tracking for studying the hematopoietic reconstitution dynamics upon HSCT

To date, few cutting-edge studies have exploited InS retrieval from GT-treated patients allowing for the first time to study the complexity of hematopoietic system and hematopoietic reconstitution upon HSCT in humans (Biasco et al. 2016; Wang et al. 2010). These studies showed that transplanted gene-repaired HSC are able to engraft and to generate polyclonal multi-lineage output overtime. Longitudinal analyses allowed unveiling that unilineage clones active during the first 6 months after GT tend to be replaced by multilineage longterm clones, indicating HSC-derived activity. Finally, based on the number of InS recaptured overtime, it has been estimated that about 1 in $10^{5}-10^{6}$ infused gene-corrected cells had the potential to engraft long term. Recently our group unveiled for the first time that primitive HSPC have a distinct role in sustaining human hematopoiesis after transplantation. While MPP are more active in the early phases, long-living HSC are on top of the hematopoietic hierarchy at steady state. Importantly we found that long-term HSC, that were activated in vitro, were capable of homing and resilience upon re-infusion (Scala et al. 2018). These approaches represent a prototypical example of the power of translational studies, providing information relevant on human hematopoietic sys- tem complementing and expanding the data derived from animal models.

\subsection{From Experimental Hematology to Medical Practices and Hematopoietic Cellular Therapies}

As already stressed in this brief review, a considerable amount of knowledge has accumulated over years allowing us to understand part of the mechanisms that control HSC behavior and take advantage of this knowledge; many of these observations cross-fertilized other disciplines. A large gap however persists between the technological sophistication of research tools and the rudimentary nature of clinical grade reagents, devices, and laboratory tests. In clinical transplantation or even in the most modern forms of hematopoietic cellular therapies, stem cells remain identified as "CD34+ cells," which can at best be considered as a gross approach to stemness; functional assays are limited to clonogenic cultures in routine practice; flow cytometryactivated cell sorting barely entered the clinical field, and most cell selection procedures rely on 
immune selection with magnetic beads. Despite these limitations, and as can be seen from the content of the other chapters in this book, HSCT remains as the only example of a worldwide and widely used cell transplant procedure, with many of its underlying conceptual aspects and techniques being used to design innovative and highly personalized somatic cell therapy or gene therapy medicinal products.

\section{Key Points \\ HSC characteristics}

- Self-renewal: ability of HSC to divide maintaining their biological properties

- Multipotency: ability of HSC to generate all mature hematopoietic cell types

- Quiescence: ability of HSC to remain inactive and unresponsive to external stimuli

\section{Models of hematopoietic hierarchy}

- Classical model: HSC differentiate into discrete and homogenous populations with successive restrictions in differentiation potentials

- Functional model: HSC differentiate according to hematopoietic state (stressed vs. unperturbed hematopoiesis)

- Progressive model: HSC differentiate through a continuous rather than discrete physiological process as result of a controlled genetic and epigenetic programs

\section{Preclinical models of HSCT}

- Murine models: study of HSC homing, engraftment, lineage differentiation, and serial transplantation capacity

- Canine models: validation of conditioning regimens and assessment of MHC donor/recipient matching significance

- Nonhuman primates: evaluation of mobilization procedures, conditioning regimens, and long-term maintenance of hematopoiesis

\section{Ex vivo gene therapy}

- Integrating vectors: $\gamma$-retroviral vectors (RV) and self-inactivating (SIN)lentiviral vectors

- Genome editing: zinc-finger nucleases (ZFN), transcription activator-like effector nucleases (TALEN), and clustered, regularly interspaced, short palindromic repeat (CRISPR) nucleases

\section{References}

Aiuti A, Naldini L. Safer conditioning for blood stem cell transplants. Nat Biotechnol. 2016;34:721-3.

Antoniani C, Romano O, Miccio A. Concise review: epigenetic regulation of hematopoiesis: biological insights and therapeutic applications. Stem Cells Transl Med. 2017;6:2106-14.

Biasco L, Pellin D, Scala S, et al. In vivo tracking of human hematopoiesis reveals patterns of clonal dynamics during early and steady-state reconstitution phases. Cell Stem Cell. 2016;19:107-19.

Busch K, Rodewald HR. Unperturbed vs. posttransplantation hematopoiesis: both in vivo but different. Curr Opin Hematol. 2016;23:295-303.

Calvi LM, Link DC. The hematopoietic stem cell niche in homeostasis and disease. Blood. 2015;126:2443-52.

Cicalese MP, Ferrua F, Castagnaro L, et al. Update on the safety and efficacy of retroviral gene therapy for immunodeficiency due to adenosine deaminase deficiency. Blood. 2016;128:45-55.

Cooper JN, Young NS. Clonality in context: hematopoietic clones in their marrow environment. Blood. 2017; 130:2363-72.

Cosgun KN, Rahmig S, Mende N, et al. Kit regulates HSC engraftment across the human-mouse species barrier. Cell Stem Cell. 2014;15:227-38.

Costa G, Kouskoff V, Lacaud G. Origin of blood cells and HSC production in the embryo. Trends Immunol. 2012;33:215-23.

Crane GM, Jeffery E, Morrison SJ. Adult haematopoietic stem cell niches. Nat Rev Immunol. 2017;17:573-90.

Di Maggio N, Piccinini E, Jaworski M, et al. Toward modeling the bone marrow niche using scaffold-based 3D culture systems. Biomaterials. 2011;32:321-9.

Doulatov S, Notta F, Laurenti E, et al. Hematopoiesis: a human perspective. Cell Stem Cell. 2012;10:120-36.

Dunbar CE, High KA, Joung JK, et al. Gene therapy comes of age. Science. 2018;359:1-10.

Eaves CJ. Hematopoietic stem cells: concepts, definitions, and the new reality. Blood. 2015;125:2605-14. 
Fares I, Chagraoui J, Gareau Y, et al. Pyrimidoindole derivatives are agonists of human hematopoietic stem cell self-renewal. Science. 2014;345:1509-12.

Goodell MA, Rando TA. Stem cells and healthy aging. Science. 2015;350:1199-204.

Gottgens B. Regulatory network control of blood stem cells. Blood. 2015;125:2614-21.

Goyal S, Zandstra PW. Stem cells: chasing blood. Nature. 2015;518:488-90.

de Haan G, Lazare S. Aging of hematopoietic stem cells. Blood. 2018;131:479-87.

Höfer T, Rodewald HR. Output without input: the lifelong productivity of hematopoietic stem cells. Curr Opin Cell Biol. 2016;43:69-77.

Kaushansky K. Lineage-specific hematopoietic growth factors. N Engl J Med. 2006;354:2034-45.

Kim S, Kim N, Presson AP, et al. Dynamics of HSPC repopulation in nonhuman primates revealed by a decade-long clonal-tracking study. Cell Stem Cell. 2014; 14:473-85.

Koelle SJ, Espinoza DA, Wu C, et al. Quantitative stability of hematopoietic stem and progenitor cell clonal output in transplanted rhesus macaques. Blood. 2017;129:1448-57.

Kohn DB. Historical perspective on the current renaissance for hematopoietic stem cell gene therapy. Hematol Oncol Clin North Am. 2017;31:721-35.

Laurenti E, Göttgens B. Review from haematopoietic stem cells to complex differentiation landscapes. Nature. 2018;553:418-26.

Lu R, Neff NF, Quake SR, et al. Tracking single hematopoietic stem cells in vivo using high-throughput sequencing in conjunction with viral genetic barcoding. Nat Biotechnol. 2011;29:928-33.

Naldini L. Ex vivo gene transfer and correction for cellbased therapies. Nat Rev Genet. 2011;12:301-15.

Pouzolles M, Oburoglu L, Taylor N, et al. Hematopoietic stem cell lineage specification. Curr Opin Hematol. 2016;23:311-7.
Reinisch A, Thomas D, Corces MR, et al. A humanized bone marrow ossicle xenotransplantation model enables improved engraftment of healthy and leukemic human hematopoietic cells. Nat Med. 2016;22:812-21.

Rowe RG, Mandelbaum J, Zon LI, et al. Engineering hematopoietic stem cells: lessons from development. Cell Stem Cell. 2016;18:707-20.

Scala S, Leonardelli L, Biasco L. Current approaches and future perspectives for in vivo clonal tracking of hematopoietic cells. Curr Gene Ther. 2016;16:1-10.

Scala S, Basso-Ricci L, Dionisio F, Pellin D, Giannelli S, Salerio FA, Leonardelli L, Cicalese MP, Ferrua F, Aiuti A, Biasco L. Dynamics of genetically engineered hematopoietic stem and progenitor cells after autologous transplantation in humans. Nat Med. 2018;24(11):1683-90. https://doi.org/10.1038/ s41591-018-0195-3.

Schoedel KB, Morcos MNF, Zerjatke T, et al. The bulk of the hematopoietic stem cell population is dispensable for murine steady-state and stress hematopoiesis. Blood. 2016;128:2285-96.

Stolfi JL, Pai C-CS, Murphy WJ. Preclinical modeling of hematopoietic stem cell transplantation-advantages and limitations. FEBS J. 2016;283:1595-606.

Sykes SM, Scadden DT. Modeling human hematopoietic stem cell biology in the mouse. Semin Hematol. 2013;2:1-14.

Wagner JE, Brunstein CG, Boitano AE, et al. Phase I/II trial of stemregenin-1 expanded umbilical cord blood hematopoietic stem cells supports testing as a standalone graft. Cell Stem Cell. 2016;18:144-55.

Wang GP, Berry CC, Malani N, et al. Dynamics of genemodified progenitor cells analyzed by tracking retroviral integration sites in a human SCID-X1 gene therapy trial. Blood. 2010;115:4356-66.

Yamamoto R, Morita Y, Ooehara J, et al. Clonal analysis unveils self-renewing lineage-restricted progenitors generated directly from hematopoietic stem cells. Cell. 2013;154:1112-26.

Open Access This chapter is licensed under the terms of the Creative Commons Attribution 4.0 International License (http://creativecommons.org/licenses/by/4.0/), which permits use, sharing, adaptation, distribution and reproduction in any medium or format, as long as you give appropriate credit to the original author(s) and the source, provide a link to the Creative Commons license and indicate if changes were made.

The images or other third party material in this chapter are included in the chapter's Creative Commons license, unless indicated otherwise in a credit line to the material. If material is not included in the chapter's Creative Commons license and your intended use is not permitted by statutory regulation or exceeds the permitted use, you will need to obtain permission directly from the copyright holder.

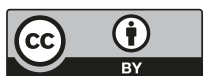

